\title{
SPICE-like Models for Nonlinear Capacitors and Inductors
}

Mihai lordache ${ }^{1}$, Lucia Dumitriu ${ }^{1}$, Mircea Perpelea ${ }^{1}$,loana-Gabriela Sîrbu², Lucian Mandache ${ }^{2}$

${ }^{1}$ Politehnica University of Bucharest, Electrical Engineering Faculty,

313 Spl. Independentei, Bucharest, Romania

iordache@elth.pub.ro,dlucia@elth.pub.ro,mircea.perpelea@bnro.ro

${ }^{2}$ University of Craiova, Electrical Engineering Faculty, 107 Decebal Boulevard,

Craiova, Romania

osirbu@elth.ucv.ro, Imandache@elth.ucv.ro

\section{ABSTRACT}

Some new models for nonlinear capacitors and nonlinear inductors built with SPICE-like element primitives are presented here. They have the advantage that they can be used for simulation in SPICE of those circuits which contain nonlinear capacitors controlled either in voltage or in charge, and nonlinear inductors controlled either in current or in flux. These SPICE-like models contain current sources whose controlling variables are charge time-derivative and sources whose controlling variables are flux time-derivative. For validation, the models are included in nonlinear circuits; their behavior is studied by simulation using two different simulators and the results are compared.

\section{Indexing terms/Keywords}

SPICE model, nonlinear capacitor, nonlinear inductor, time-domain analysis.

\section{Academic Discipline And Sub-Disciplines}

Electrical Engineering; Electric Circuits; Software Engineering; Microelectronics Engineering; Instrumentation electronics; Smart devices and intelligent environments

\section{SUBJECT CLASSIFICATION}

Library of Congress Classification

\section{TYPE (METHOD/APPROACH)}

Theoretical Study; Simulation

\section{Council for Innovative Research}

Peer Review Research Publishing System

Journal: INTERNATIONAL JOURNAL OF COMPUTERS \& TECHNOLOGY

Vol 12, No.2

editor@cirworld.com

www.cirworld.com, member.cirworld.com 


\section{INTRODUCTION}

SPICE is one of the most well-known electrical circuit simulators, being used not only in the educational or research activities, but also in industry, for computer-aided circuit design of analog circuits [1-19]. At the same time, as a generally accepted recognition, it represented also the basis for the development of new software packages used for the simulation of the electric and electronic circuits.

These programs meet a continuous development to correspond to the new requirements demanded by the customer. One of the resources that had a permanent change concerning its extension was the components' library of the programs [19]. Thus, if the initial programs were simple, containing only the basic elements of the electric circuits, today the software library is extremely complex, including the electronic components of some well-known manufacturers, but also elements corresponding to some complex circuit structures with practical applicability. In this way the costs of acquiring a SPICEtype software package increased significantly.

However, if a particular circuit element is not met in the library of the SPICE-type software, it has to be built with existing primitive elements from that library. Recent works tackle this subject, offering solutions for the modeling of new circuit elements or of parts of some electric systems [2], [4], [14]-[17].

A special attention was paid to the nonlinear circuit elements, such as the nonlinear inductor and the nonlinear capacitor [2], [4], [6], [11], [13]. For a nonlinear inductor with a current dependent inductance or a nonlinear capacitor with a voltage dependent capacitance the characteristics specification by using a look-up table in the ABM (Analog Behavioral Modelling) or by using a polynomial representation of the second or third order whose coefficients can be defined in a .MODEL statement were proposed.

In this paper we propose SPICE-like models for nonlinear capacitors controlled either in voltage or in charge, and nonlinear inductors controlled either in current or in flux. In order to be validated, these models are implemented in electric circuits and their behavior is analyzed by using two different software packages: SPICE - as commercial software, and ENCAP (Electrical Nonlinear Circuit Analysis Program) - our original program for analog circuit analysis [8]. The proposed models contain either voltage sources whose controlling variables are current time-derivatives (CTDCVS) or current sources whose controlling variables are voltage time-derivatives (VTDCCS). For this reason we firstly present the models of these controlled sources (undefined in SPICE) that uses equivalent circuits containing only primitive circuit elements accepted by SPICE.

\section{MODEL FOR THE CURRENT TIME-DERIVATIVE CONTROLLED VOLTAGE SOURCES}

A voltage source whose controlling variable is a current time-derivative, Figure 1 (a) and Figure 2 (a), can be modeled either by a voltage-controlled voltage source, as in Figure 1 (b), or by a current-controlled voltage source, as in Figure 2 (b). In both cases the modeling is based on an exchange of variable.

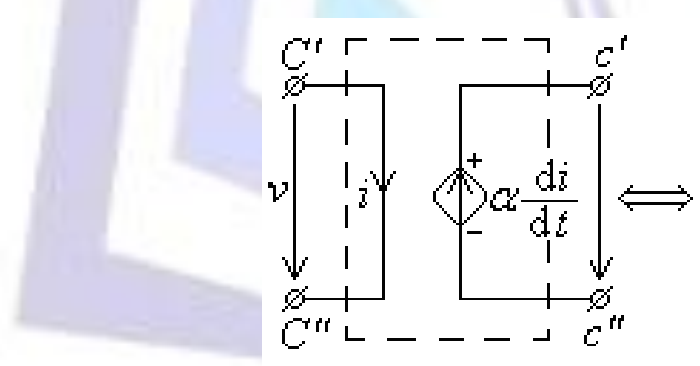

(a)

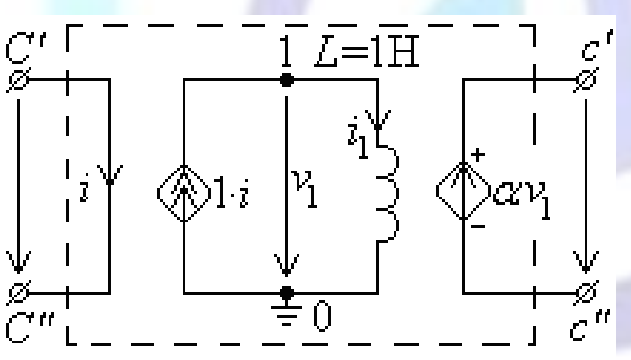

(b)

Fig 1: CTDCVS modeled by SPICE-like element primitives and voltage-controlled voltage source

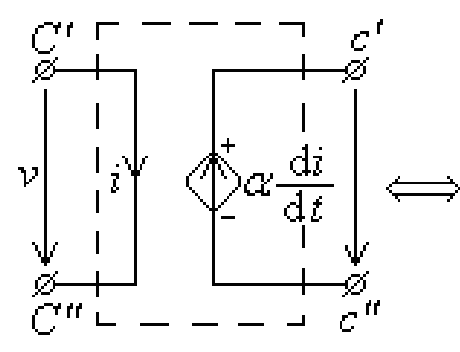

(a)

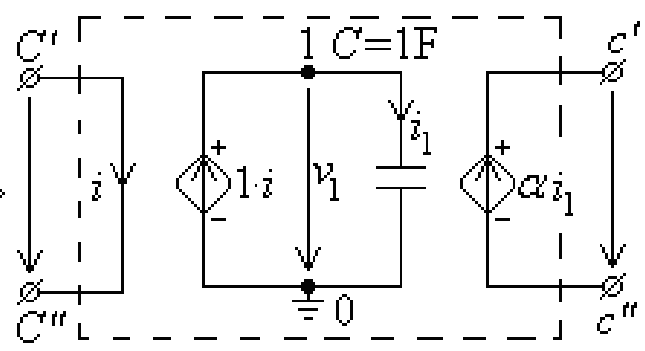

(b)

Fig 2: CTDCVS modeled by SPICE-like element primitives and current-controlled voltage source 
The first model, presented in Figure 1, is based on an intermediate circuit containing a current-controlled current source and an inductor; the voltage across the inductor, $v_{1}$, changes the controlling variable from a current time-derivative in a voltage. The transformation is based on the following equations [14]:

$$
\begin{aligned}
& j_{c}(i)=1 \cdot i=i_{1} \\
& v_{1}=L \frac{\mathrm{d} i_{1}}{\mathrm{~d} t}=\frac{\mathrm{d} i_{1}}{\mathrm{~d} t}=\frac{\mathrm{d} i}{\mathrm{~d} t}
\end{aligned}
$$

The second model, presented in Figure 2, is based on an intermediate circuit containing a current-controlled voltage source and a capacitor; the current through the capacitor, $i_{1}$, changes the controlling variable from a voltage timederivative in a current. The involved equations are:

$$
\begin{aligned}
& e_{c}(i)=1 \cdot i=v_{1} \\
& i_{1}=C \frac{\mathrm{d} v_{1}}{\mathrm{~d} t}=\frac{\mathrm{d} i}{\mathrm{~d} t}
\end{aligned}
$$

\section{MODEL FOR THE VOLTAGE TIME-DERIVATIVE CONTROLLED CURRENT SOURCES}

A current source whose controlling variable is a voltage time-derivative, Figure 3 (a) and Figure 4 (a), can be modeled either by a voltage-controlled current source, as in Figure 3 (b), or by a current-controlled current source, as in Figure 4 (b). In both cases the modeling is also based on an exchange of variable.

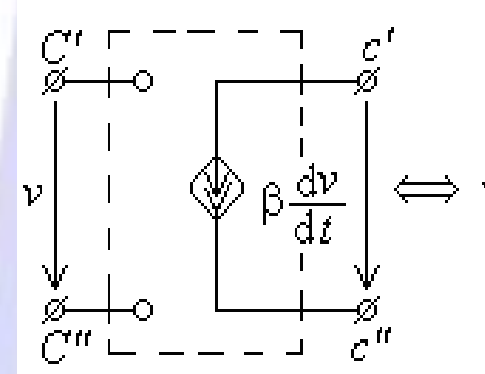

(a)

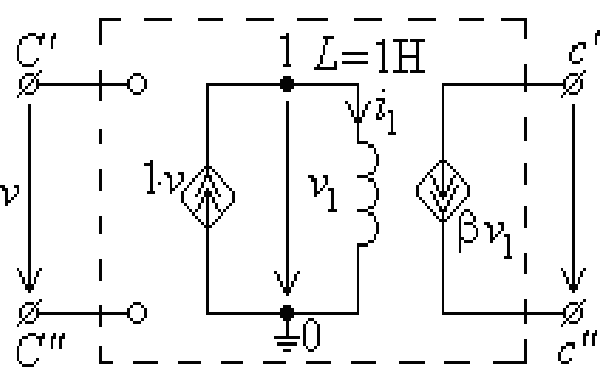

(b)

Fig 3: VTDCCS modeled by SPICE-like element primitives and voltage-controlled current source

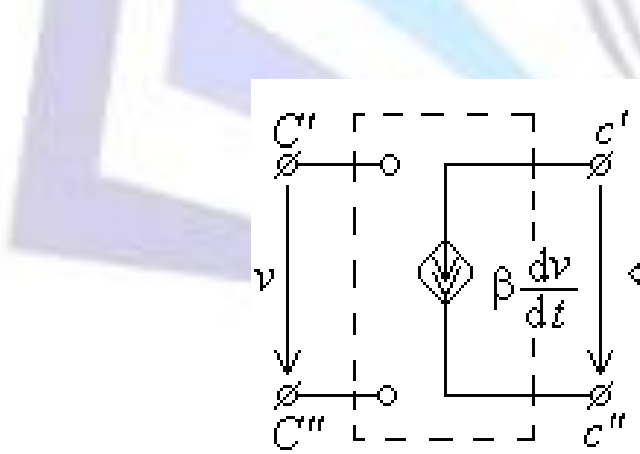

(a)

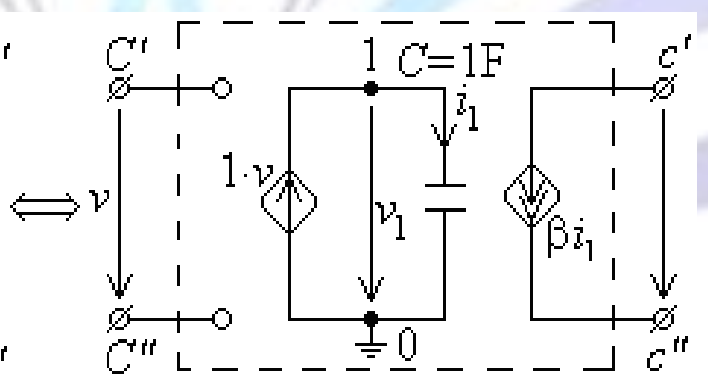

(b)

Fig 4: VTDCCS modeled by SPICE-like element primitives and current-controlled current source

The first model, presented in Figure 3 , is based on an intermediate circuit containing a voltage-controlled current source and an inductor; the voltage across the inductor, $v_{1}$, changes the controlling variable from a voltage time-derivative in a voltage. The transformation is based on the following equations [14]:

$$
\begin{aligned}
& j_{c}(v)=1 \cdot v=i_{1} \\
& v_{1}=L \frac{\mathrm{d} i_{1}}{\mathrm{~d} t}=\frac{\mathrm{d} i_{1}}{\mathrm{~d} t}=\frac{\mathrm{d} v}{\mathrm{~d} t}
\end{aligned}
$$


The second model, presented in Figure 4, is based on an intermediate circuit containing a voltage-controlled voltage source and a capacitor; the current through the capacitor, $i_{1}$, changes the controlling variable from a voltage timederivative in a current. The involved equations are:

$$
\begin{aligned}
& e_{c}(v)=1 \cdot v=v_{1} \\
& i_{1}=C \frac{\mathrm{d} v_{1}}{\mathrm{~d} t}=\frac{\mathrm{d} v}{\mathrm{~d} t}
\end{aligned}
$$

\section{SPICE-LIKE MODEL FOR A VOLTAGE CONTROLLED NONLINEAR CAPACITOR}

In order to obtain a SPICE-like model for a voltage-controlled (v.c.) nonlinear capacitor (circuit element that is not defined in SPICE) we have to transfer the constitutive equation

$$
i=\frac{\mathrm{d} q}{\mathrm{~d} t}=\frac{\mathrm{d} q}{\mathrm{~d} v} \cdot \frac{\mathrm{d} v}{\mathrm{~d} t}=C_{d}(v) \frac{\mathrm{d} v}{\mathrm{~d} t}
$$

to a controlled current source whose current obeys the equation (5). In the same time the nonlinear characteristic that gives the charge dependence of the voltage, $q=\hat{q}(v)$, will be transferred to a SPICE compatible nonlinear element. To this end a nonlinear resistor $G_{n l}$ is used; its nonlinear characteristic $i_{2}=\hat{i}_{2}\left(v_{2}\right)$ reproduces the characteristic $q=\hat{q}(v)$ of the nonlinear capacitor. In this way the charge is converted into the nonlinear resistor current. In order to keep the correspondence between the voltage variables we need to introduce a voltage-controlled voltage source in the nonlinear resistor circuit. Thus the following relation can be written:

$$
v_{2}=E_{2}=1 \cdot v
$$

To return to the initial variable (charge $q$ ) a linear capacitor is used like in the model developed in Figure 2 . The following relations are valid in the corresponding subcircuit:

$$
\begin{aligned}
& v_{3}=H_{3}=1 \cdot i_{c 2}=1 \cdot i_{2} \\
& i_{3}=C \frac{\mathrm{d} v_{3}}{\mathrm{~d} t}=1 \cdot \frac{\mathrm{d} i_{2}}{\mathrm{~d} t}=\frac{\mathrm{d} q}{\mathrm{~d} t}
\end{aligned}
$$

If the capacitor current $i_{3}=i_{c 3}$ is taken as controlling variable of a current-controlled current source, we get the SPICElike model presented in Figure 5 [14]. We can remark that the model contains only circuit elements admitted by SPICE.

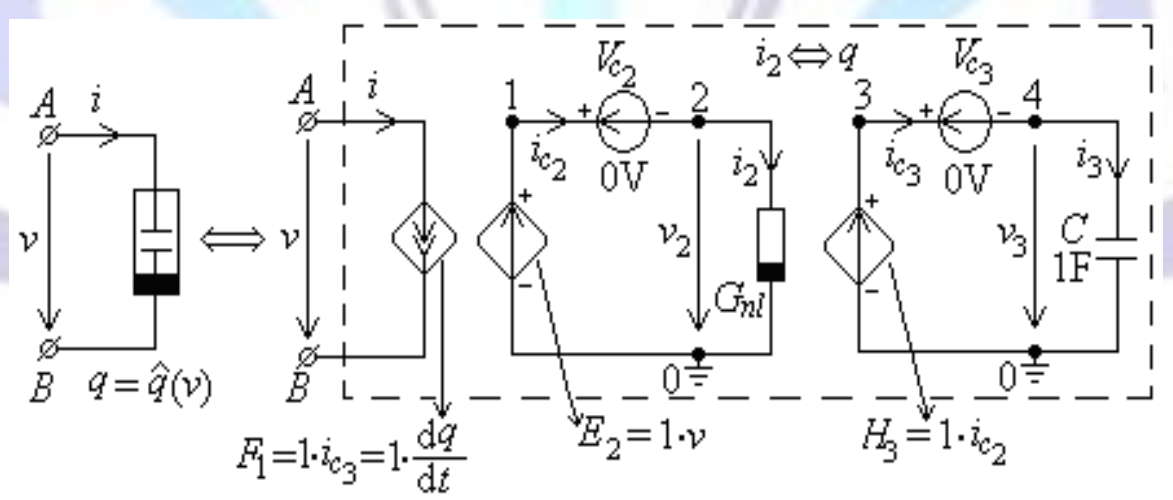

Fig 5: SPICE-like model of a voltage-controlled nonlinear capacitor

\section{Remark}

The reference node $(0)$ can be any of the circuit nodes (even the reference node of the general circuit). The other nodes, $1,2,3$ and 4 , can be numbered as $n+1, n+2, n+3$ and $n+4$ ( $n$ being the total number of the circuit nodes).

\section{SPICE-LIKE MODEL FOR A CHARGE CONTROLLED NONLINEAR CAPACITOR}

The SPICE-like model of a charge-controlled (q.c.) nonlinear capacitor can be obtained by using a nonlinear resistor and a linear inductor, as in Figure 6 [14]. The variable exchange is accomplished by a voltage-controlled current source, as in Figure 3. 


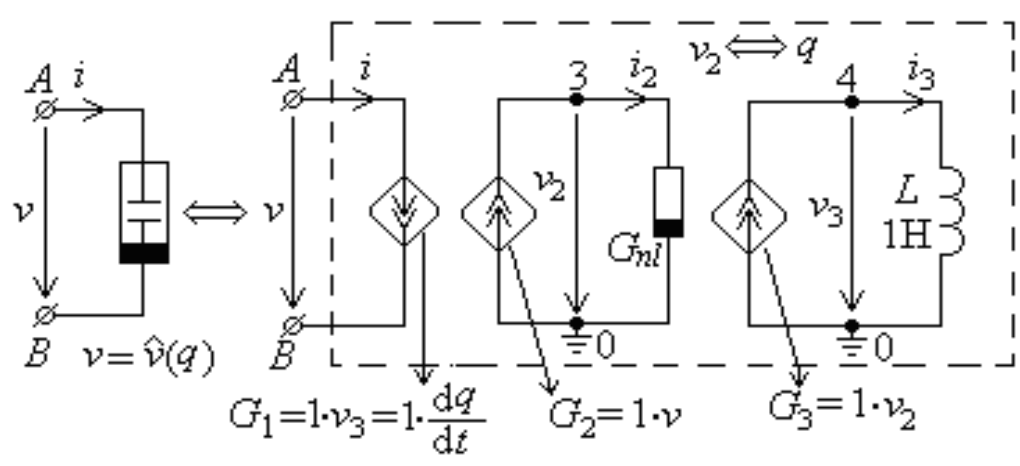

Fig 6: SPICE-like model of a charge controlled nonlinear capacitor

The nonlinear characteristic $v=\hat{v}(q)$ of the nonlinear capacitor is transferred as $i_{2}=\hat{i}_{2}\left(v_{2}\right)$ to the nonlinear resistor $G_{n l}$. In this way the charge is converted into the nonlinear resistor voltage and the voltage into the nonlinear resistor current by means of a voltage-controlled current source. The linear inductor allows restoring the initial variable (charge $q$ ). The following relations can be written [14]:

$$
i_{2}=G_{2}=1 \cdot v
$$

and

$$
\begin{aligned}
& i_{3}=G_{3}=1 \cdot v_{2}=1 \cdot q \\
& v_{3}=L \frac{\mathrm{d} i_{3}}{\mathrm{~d} t}=1 \cdot \frac{\mathrm{d} v_{2}}{\mathrm{~d} t}=\frac{\mathrm{d} q}{\mathrm{~d} t} .
\end{aligned}
$$

If the inductor voltage $v_{3}$ is taken as controlling variable of a voltage-controlled current source, we get the SPICE-like model presented in Figure 6 . Thus the model contains only circuit elements admitted by SPICE.

\section{Remark}

The reference node (0) can be any of the circuit nodes (including the reference node of the general circuit). The other nodes, 3 and 4 , can be numbered as $n+3$ and $n+4$ ( $n$ being the total number of the circuit nodes).

\section{SPICE-LIKE MODEL FOR A CURRENT CONTROLLED NONLINEAR INDUCTOR}

In order to generate a SPICE-like model for a current-controlled (c.c.) nonlinear inductor (circuit element that is not defined in SPICE) we have to transfer the characteristic equation

$$
v=\frac{\mathrm{d} \varphi}{\mathrm{d} t}=\frac{\mathrm{d} \varphi}{\mathrm{d} i} \cdot \frac{\mathrm{d} i}{\mathrm{~d} t}=L_{d}(i) \frac{\mathrm{d} i}{\mathrm{~d} t}
$$

to a controlled voltage source whose voltage obeys the equation (10). In the same time the nonlinear characteristic that gives the magnetic flux dependence of the current, $\varphi=\hat{\varphi}(i)$, will be transferred to a SPICE compatible nonlinear element. To this end a nonlinear resistor $G_{n l}$ is used; its nonlinear characteristic $i_{2}=\hat{i}_{2}\left(v_{2}\right)$ reproduces the characteristic $\varphi=\hat{\varphi}(i)$ of the nonlinear inductor ( $i_{2} \Leftrightarrow \varphi$ and $v_{2} \Leftrightarrow i$ ). In this way the magnetic flux is converted into the nonlinear resistor current. In order to keep the correspondence between the electrical variables we need to introduce a current-controlled voltage source in the nonlinear resistor circuit. Thus the following relation can be written:

$$
v_{2}=H_{2}=1 \cdot i_{c 1} \text {. }
$$

To return to the initial variable (flux $\varphi$ ) a linear capacitor is used like in the model developed in Figure 2. The following relations are valid in the corresponding subcircuit:

$$
\begin{aligned}
& v_{3}=H_{3}=1 \cdot i_{c 2}=1 \cdot i_{2} \\
& i_{c 3}=i_{C}=C \frac{\mathrm{d} v_{C}}{\mathrm{~d} t}=1 \cdot \frac{\mathrm{d} i_{2}}{\mathrm{~d} t}=\frac{\mathrm{d} \varphi}{\mathrm{d} t} .
\end{aligned}
$$

If the capacitor current $i_{3}=i_{c 3}$ is taken as controlling variable of a current-controlled voltage source, we get the SPICElike model presented in Figure 7. We can remark that the model contains only circuit elements admitted by SPICE. 


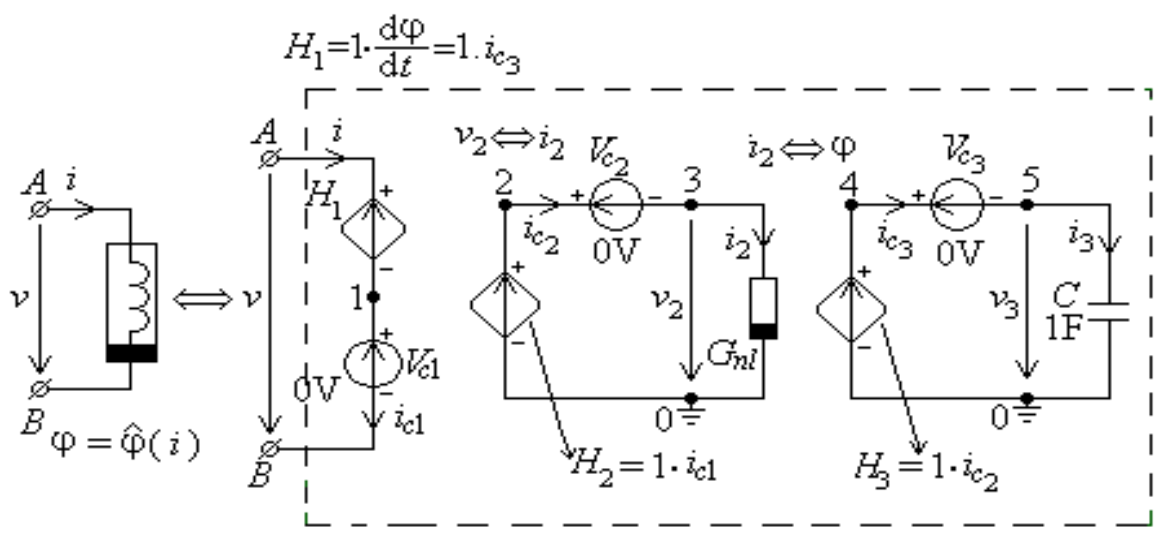

Fig 7: SPICE-like model of a current controlled nonlinear inductor

\section{Remark}

The reference node $(0)$ can be any of the circuit nodes (even the reference node of the general circuit). The other nodes, 1, 2, 3, 4 and 5, can be numbered as $n+1, n+2, n+3, n+4$ and $n+5$ ( $n$ being the total number of the circuit nodes).

\section{SPICE-LIKE MODEL FOR A FLUX CONTROLLED NONLINEAR INDUCTOR}

The SPICE-like model of a flux-controlled ( $\varphi$. c.) nonlinear inductor can be obtained by using a nonlinear resistor and a linear inductor, as in Figure 8. The variable exchange is accomplished by a voltage-controlled voltage source, as in Figure 1 .

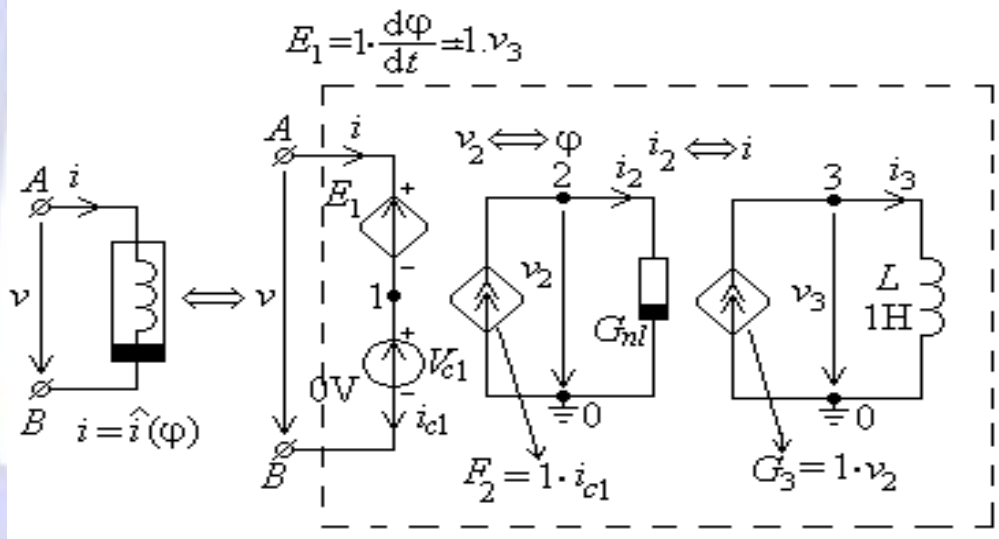

Fig 8: SPICE-like model of a flux controlled nonlinear inductor

The nonlinear characteristic $i=\hat{i}(\varphi)$ of the flux-controlled nonlinear inductor is transferred as $i_{2}=\hat{i}_{2}\left(v_{2}\right)$ to the nonlinear resistor $G_{n l}$. In this way the flux is converted into the nonlinear resistor voltage and the current into the nonlinear resistor current by means of a current-controlled current source. The linear inductor allows restoring the initial variable (flux $\varphi$ ). The following relations can be written:

$$
i_{2}=F_{2}=1 \cdot i_{c 1}=1 \cdot i
$$

and

$$
\begin{aligned}
& i_{3}=G_{3}=1 \cdot v_{2}=1 \cdot \varphi \\
& v_{3}=L \frac{\mathrm{d} i_{3}}{\mathrm{~d} t}=1 \cdot \frac{\mathrm{d} v_{2}}{\mathrm{~d} t}=\frac{\mathrm{d} \varphi}{\mathrm{d} t} .
\end{aligned}
$$

If the inductor voltage $v_{3}$ is taken as controlling variable of a voltage-controlled voltage source, we get the SPICE-like model presented in Figure 8. Thus the model contains only circuit elements admitted by SPICE.

\section{Remark}

The reference node $(0)$ can be any of the circuit nodes (including the reference node of the general circuit). The other nodes, 1, 2 and 3 , can be numbered as $n+1, n+2$ and $n+3$ ( $n$ being the total number of the circuit nodes). 


\section{EXAMPLES}

\subsection{Voltage-controlled nonlinear capacitor}

Let us consider the circuit in Figure 9 containing a voltage-controlled nonlinear capacitor. The nonlinear characteristic $q_{C_{4}}=\hat{q}_{C_{4}} \boldsymbol{}_{C_{4}}$, of the voltage-controlled nonlinear capacitor has the following expression:

$$
q_{C_{4}}=5.0 \cdot v_{C_{4}}+20.0 \cdot v_{C_{4}}^{3} .
$$

The two ideal independent voltage sources $e_{1}$ and $e_{2}$ are the sinusoidal signals of diferit frequences:

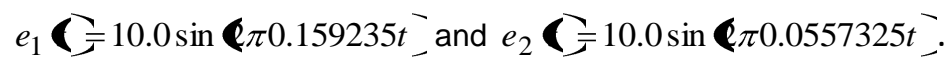

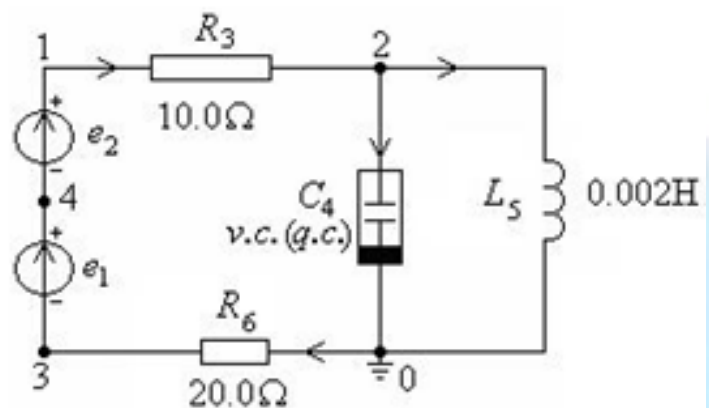

Fig 9: Circuit with a voltage-controlled (charge-controlled) nonlinear capacitor

Running SPICE by using the model of the nonlinear capacitor from Figure 5 we get the voltage across the nonlinear capacitor as in Figure 10.

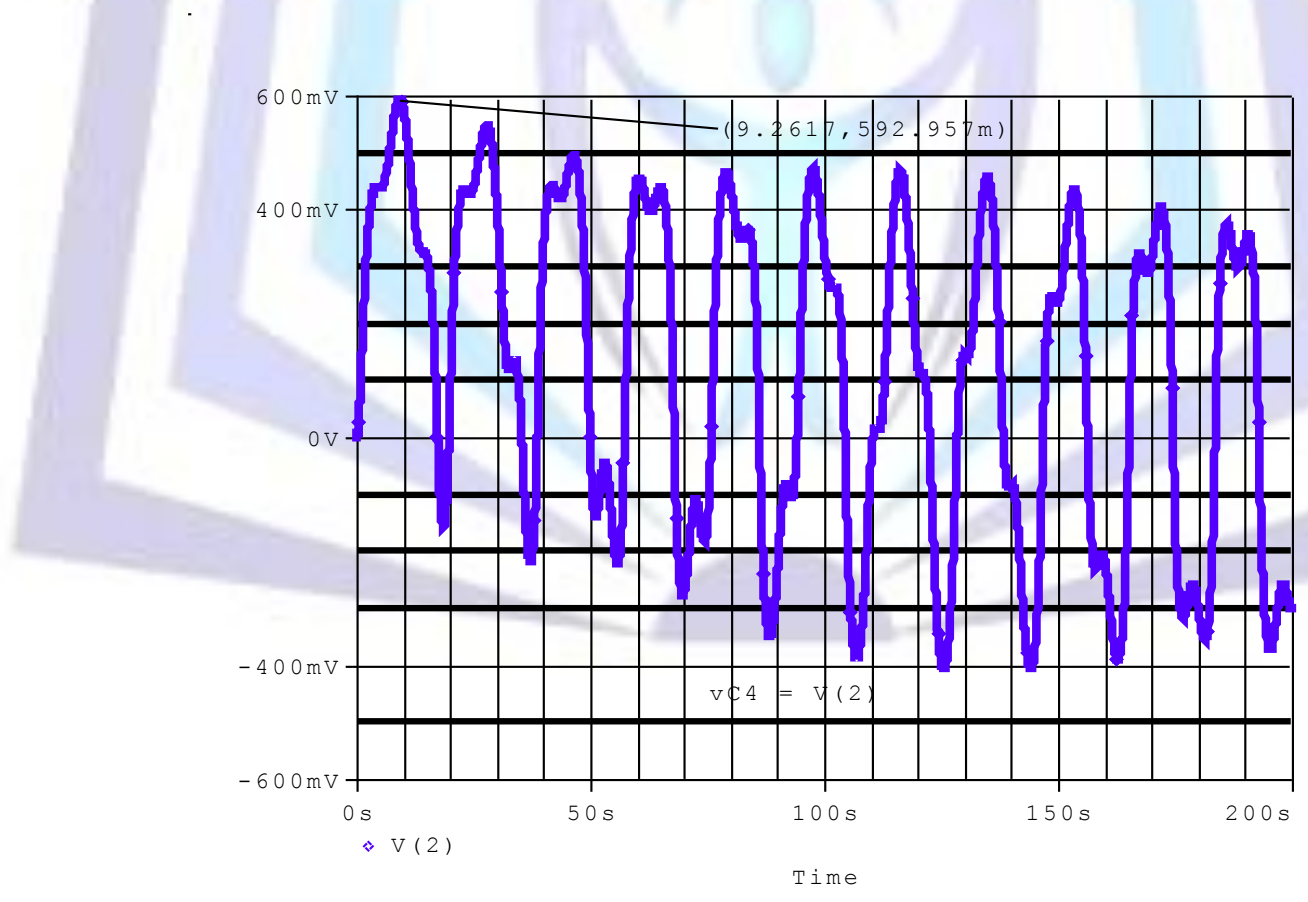

Fig 10: Time-variation of the v.c. nonlinear capacitor voltage by SPICE

The same result is obtained by using our original program - ENCAP [8] - in which the two models were implemented (Figure 11). 


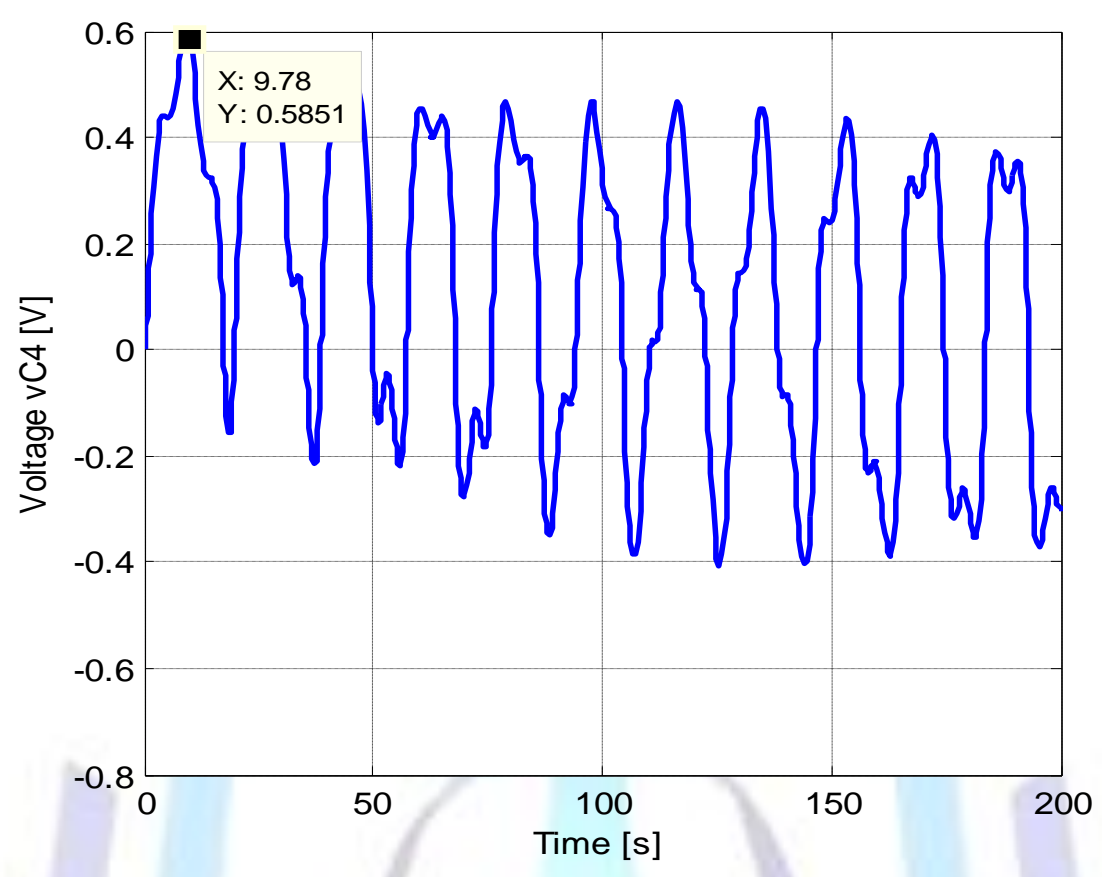

Fig 11: Time-variation of the v.c. nonlinear capacitor voltage by ENCAP

\subsection{Charge-controlled nonlinear capacitor}

We solve the circuit in Figure 9 considering that the nonlinear capacitor is charge-controlled (q.c.). In this case the nonlinear characteristic $v_{C_{4}}=\hat{v}_{C_{4}}{ }_{C_{4}}$, has the following expression:

$$
{ }^{v_{C_{4}}}=5.0 \cdot q_{C_{4}}+20.0 \cdot q_{C_{4}}^{3} .
$$

The results obtained with SPICE and ENCAP are presented in Figure 12 and Figure 13, respectively.

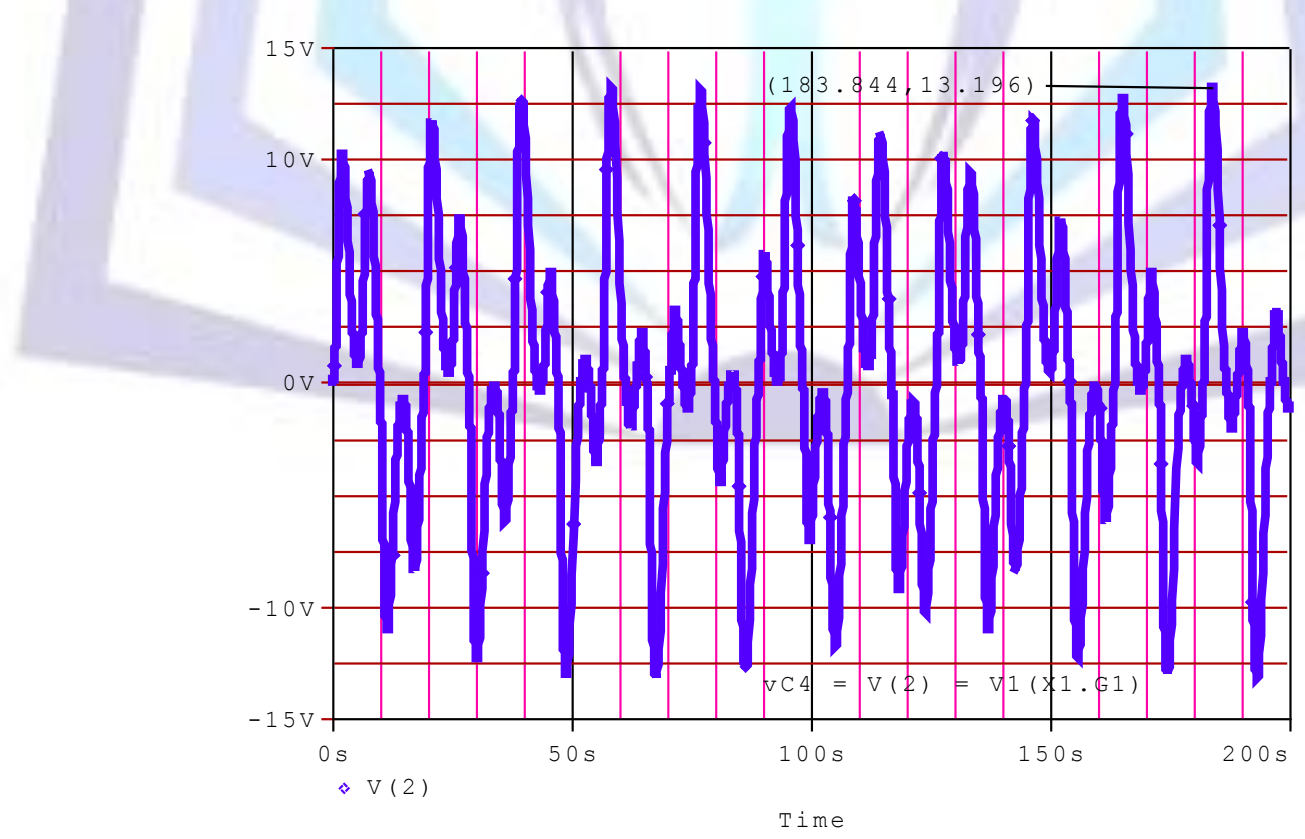

Fig 12: Time-variation of the q.c. nonlinear capacitor voltage by SPICE 


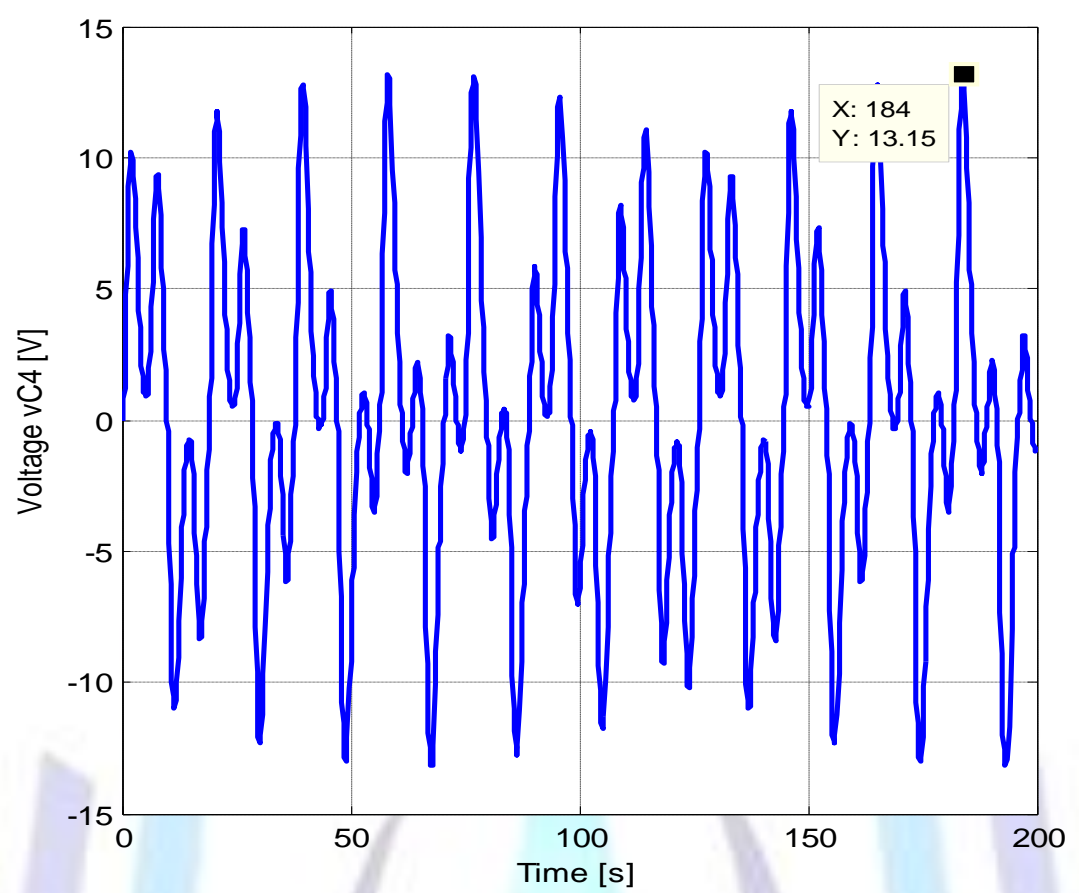

Fig 13: Time-variation of the q.c. nonlinear capacitor voltage by ENCAP The results obtained with the two simulators are very closed.

\subsection{Current-controlled nonlinear inductor}

We consider the nonlinear circuit in Figure 14 containing a current-controlled nonlinear inductor.

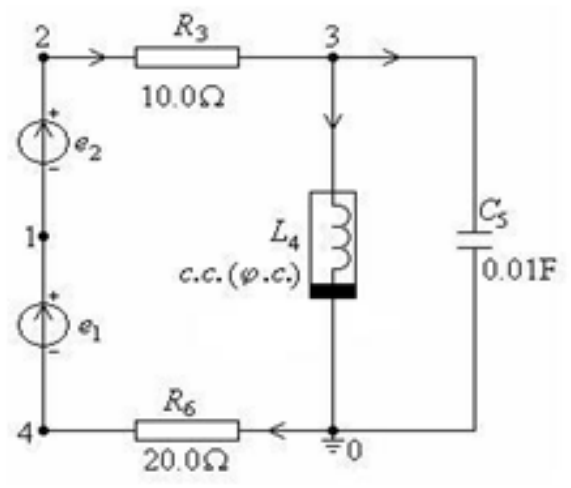

Fig 14: Circuit with a current-controlled (flux-controlled) nonlinear inductor

The nonlinear characteristic $\varphi_{L_{4}}=\hat{\varphi}_{L_{4}} \mathbf{L}_{4}$, of the current-controlled (c.c.) nonlinear inductor has the following expression:

$$
\varphi_{L_{4}}=10 \cdot i_{L_{4}}+50 \cdot i_{L_{4}}^{3}
$$

The two ideal independent voltage sources $e_{1}$ and $e_{2}$ are the sinusoidal signals of diferit frequences with the expression given in (16).

Running SPICE by using the model of the current-controlled nonlinear inductor in Figure 7 we get the current through the nonlinear inductor as in Figure 15. 


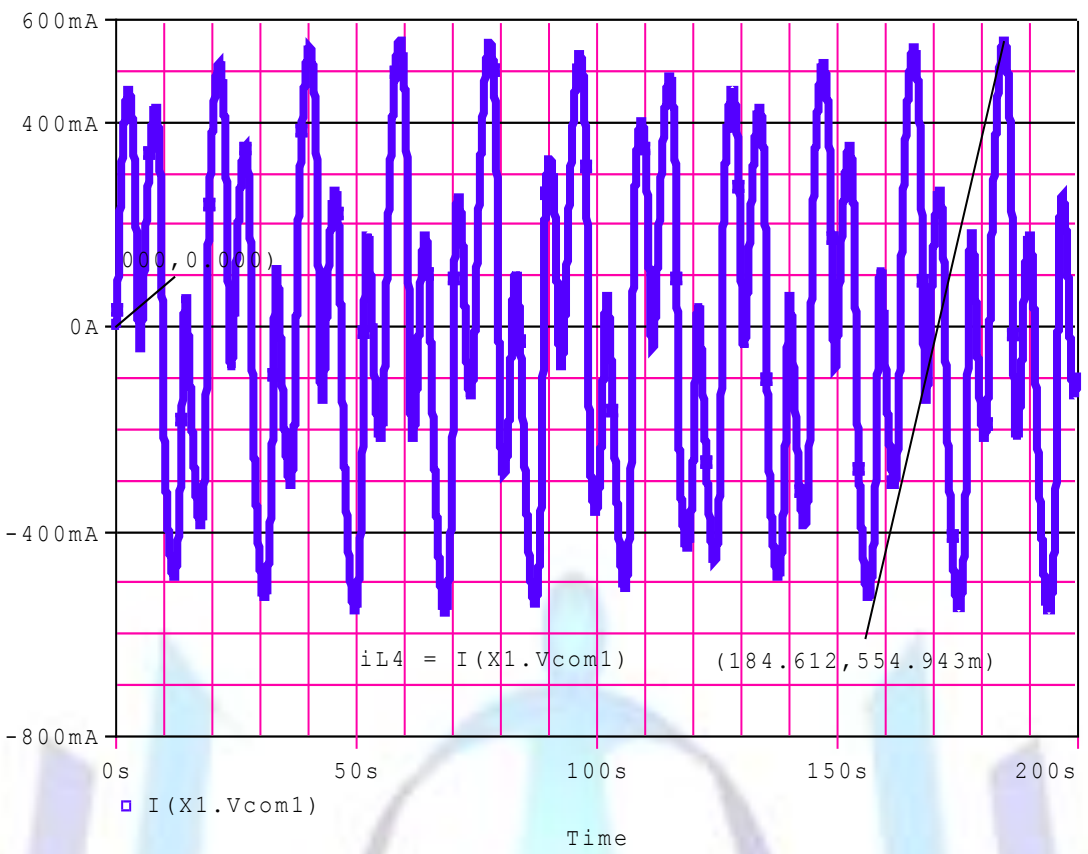

Fig 15: Time-variation of the c.c. nonlinear inductor current by SPICE The same result is obtained by ENCAP (Figure 16).

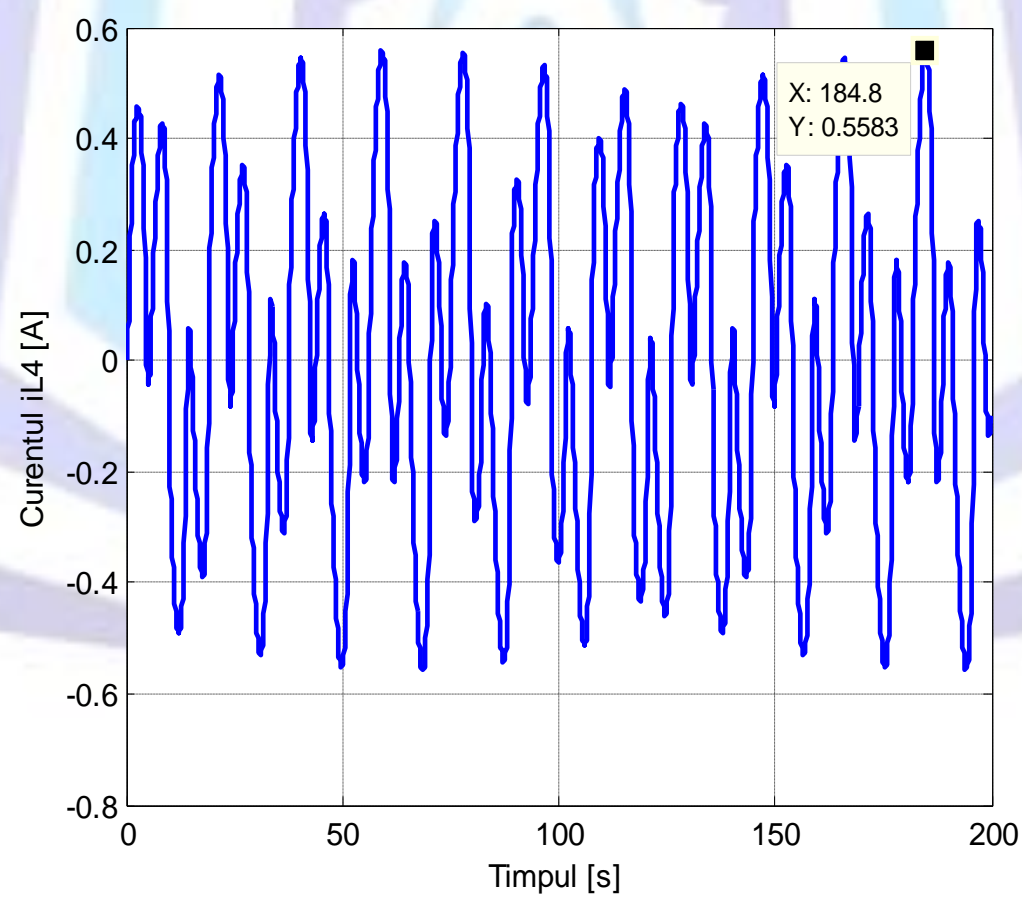

Fig 16: Time-variation of the c.c. nonlinear inductor current by ENCAP

\subsection{Flux-controlled nonlinear inductor}

We solve the circuit in Figure 14 considering that the nonlinear inductor is flux-controlled ( $\varphi$.c.). In this case the nonlinear characteristic $i_{L_{4}}=\hat{i}_{L_{4}} \boldsymbol{Q}_{L_{4}}$, has the following expression:

$$
i_{L_{4}}=5.0 \cdot \varphi_{L_{4}}+20.0 \cdot \varphi_{L_{4}}^{3} .
$$

The results obtained with SPICE and ENCAP are presented in Figure 17 and Figure 18, respectively. 


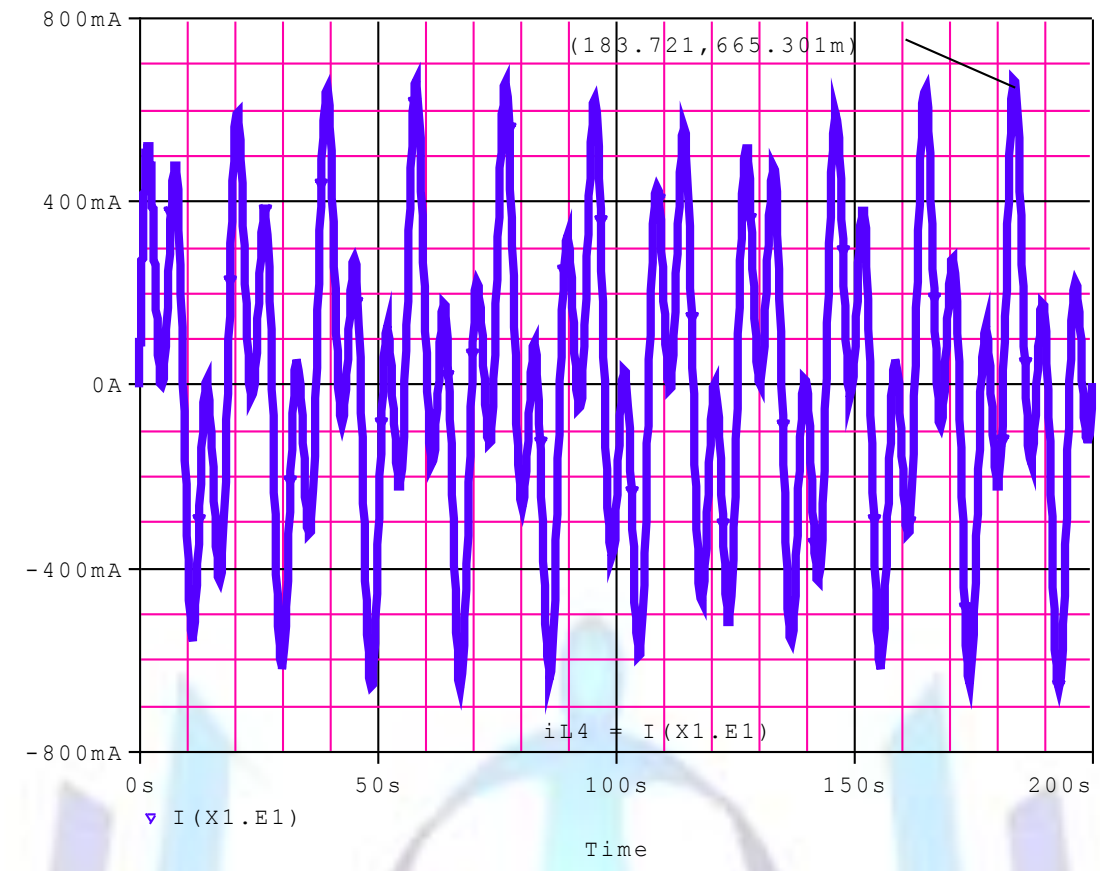

Fig 17: Time-variation of the $\varphi . c$. nonlinear inductor current by SPICE

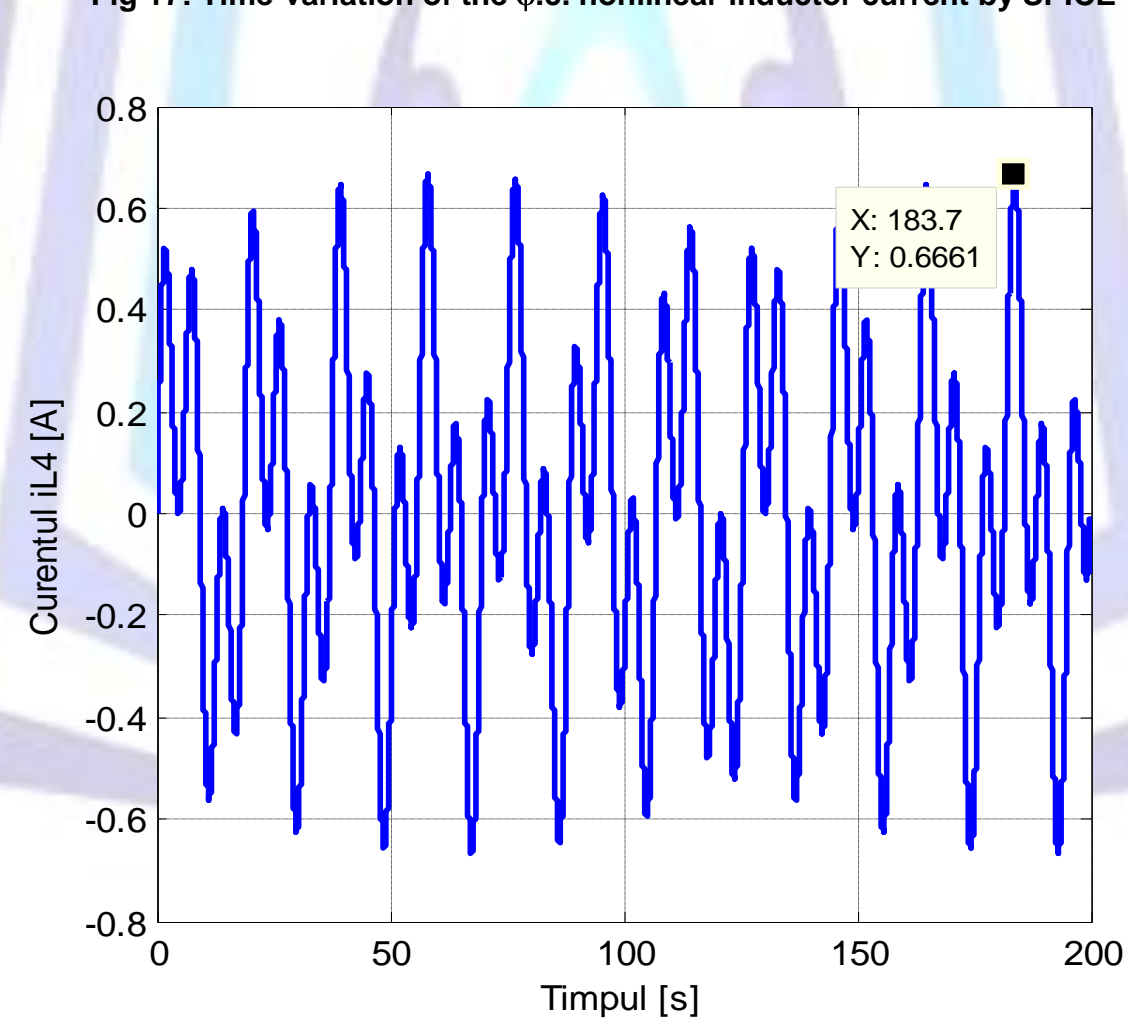

Fig 18: Time-variation of the $\varphi$.c. nonlinear inductor current by ENCAP

We can remark the good agreement between the results obtained with the two simulators.

\section{CONCLUSION}

The most used analog circuit simulator (SPICE) has a rich library of electric and electronic elements. However certain type of nonlinear elements, such as nonlinear capacitor and nonlinear inductor, are not fully completed since the simulator does not include nonlinear capacitor both voltage and charge controlled and nonlinear inductor both current and flux controlled. For this reason we built SPICE-like models for these circuit elements using only SPICE compatible elements.

The proposed models contain voltage sources whose controlling variables are current time-derivatives or current sources whose controlling variables are voltage time-derivatives. Because these kind of controlled sources are not defined in 
SPICE, firstly we modeled them by equivalent circuits containing only primitive circuit elements accepted by the standard simulator.

The validation of the models was done by simulations performed both in SPICE and in an original analysis program ENCAP - in which these models were implemented.

\section{REFERENCES}

[1] SPICE tutorial. MICROELECTRONICS. Introduction to SPICE, A brief overview. www.brunel.ac.uk/ eestmba/usergS.html.

[2] A Nonlinear Capacitor Model for Use in the PSpice Environment. Application Note. Cadence, December 2009.

[3] Plett, C. SPICE 1 modified user's guid. Department of Electronics, Carleton University.

[4] Biolek, D., Kolka, Z., and Biolkova, V. 2007 Modeling time-varying storage components in PSpice. http://user.unob.cz/biolek/veda/articles/EDS07.pdf.

[5] Rashid, M.H 1990. SPICE for Circuits and Electronics Using Pspice. Prentice-Hall International Editions.

[6] Oksasoglu, A., and Huelsman, L. P. Interaction of capacitive and resistive nonlinearities in Chua's circuit. In Engineering Analog Integrated Circuits and Signal Processing 12, 3, 239-253.

[7] Design Simulation and Device Models, Linear Technology. 2010. http://www.linear.com.

[8] Iordache, M., Dumitriu, Lucia, and Matei, I. 2001. ENCAP - Electrical Nonlinear Circuit Analysis Program. User Guide. Department Electrical Library, Politehnica University of Bucharest.

[9] Brambilla, A., and Maffezzoni, P. 2003. Envelope-following method to compute steady-state solutions of electric circuits. IEEE Transactions on Circuits and Systems - I: Fundamental Theory and Applications 50 (March 2003), 407417.

[10] Chua, L.O. , and Lin, P.M. 1975. Computer-Aided Analysis of Electronic Circuits - Algorithms and Computational Techniques. Prentice-Hall, Inc. Englewood Cliffs, New Jersey.

[11] Aprille, T.J. , and Trick, T.N. 1972. Steady-state analysis of nonlinear circuits with periodic inputs. In Proc. IEEE 60 (Jan. 1972), 108-114.

[12] Schwarz, A. F. 1987. Computer-Aided Design of Microelectronic Circuits and Systems. Fundamentals, Methods and Tools. Vol. 1 - General Introduction and Analog-Circuit Aspects. Academic Press, New York.

[13] Gheorghe, A. G., and Constantinescu, F. 2010. Solution computation for resistive circuits containing companion models. In U.P.B. Scientific Bulletin, 72, 4, 193-204.

[14] Dumitriu, Lucia, Iordache, M., Mandache, L., Sirbu, loana-Gabriela, and Dumitrescu, lulia 2011. SPICE-like models for nonlinear capacitors. In Acta Electrotehnica Proceedings of the 4nd International Conference on Modern Power Systems, MPS 2011. Mediamira Science Publisher, 151 - 154.

[15] Mandache, L., Topan, D., Iordache, M., and Sirbu, I.G. 2013. SPICE model for fast time domain simulation of power transformers, exploiting the ferromagnetic hysteresis and eddy-currents. Selected Topics in Nonlinear Dynamics and Theoretical Electrical Engineering. Studies in Computational Intelligence 459, 273-286.

[16] Hsu, J.T., and Ngo, K.D.T. 2002. Subcircuit modeling of magnetic cores with hysteresis in PSpice. IEEE Transactions on Aerospace and Electronic Systems 38, 4 (October 2002), 1425-1434.

[17] Sîrbu, I.G., Mandache L., and lordache M. 2011. A novel model of dc series motor based on electrical equivalent circuit. In Proceedings of the International Symposium on Signals, Circuits and Systems ISSCS.

[18] Antonini, G. , Sabatini, M. , and Miscione, G. 2006. PEEC modeling of linear magnetic materials. In Proceedings of IEEE International Symposium on Electromagnetic Compatibility EMC, 93 - 98.

[19] Urquia, A., Martin, C., and Dormido, S. 2005. Design of SPICELib: A Modelica library for modeling and analysis of electric circuits. Mathematical and Computer Modelling of Dynamical Systems 11 (March 2005), 43 - 60.

\section{Authors' biographies with Photos}

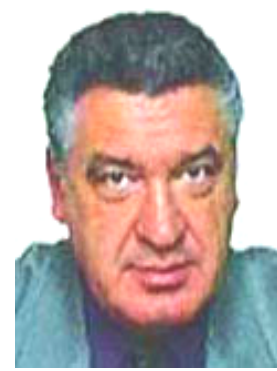

Mihai lordache received the M.S. and Ph.D. degrees in electrical engineering from the Politehnica University of Bucharest, Romania, in 1967 and 1977, respectively. He is a Full Professor in the Electrical Department, Politehnica University of Bucharest, where he is working in the areas of circuit analysis and simulation, and in the Electrical Engineering Fundamentals. He is Doctoral Advisor at the Politehnica University of Bucharest, and the author or coauthor of more than 180 journal papers and 25 books. He is also a reviewer of different Scientific Conferences in the Analysis and Simulation Circuits. He was the recipient of the 2000 Romanian Academy Award. 

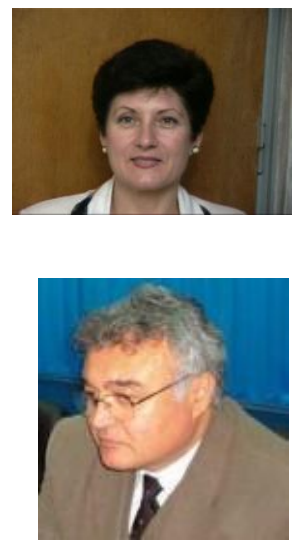

Lucia Dumitriu received the B.S. and Ph.D. degrees in electrical engineering from Politehnica University of Bucharest, Romania, in 1974 and 1987, respectively. She is currently a full Professor in the Electrical Department, Politehnica University of Bucharest, teaching and doing research on Electrical Engineering Fundamentals, and Circuit Theory. Her research interests include analysis and simulation of analog circuits, symbolic analysis, and computer-aided design of large-scale circuits. She was the recipient of the 2000 Romanian Academy Award.

Mircea Perpelea received the M.S. degree in electrical engineering from the University of Craiova, Romania and the Ph.D. degree from the Politehnica University of Bucharest, in 1997. He is currently an associated Professor at the University of Pitesti, Romania, teaching and doing research on power energy, the use of the electrical energy, and electrical equipments.

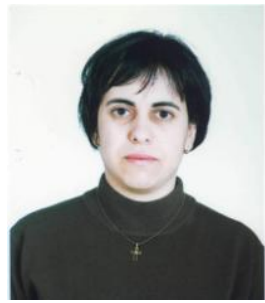

Ioana-Gabriela Sîrbu received the M.S. degree in electrical engineering from University of Craiova in 2000 and the Ph.D. degree in electrical engineering from Politehnica University of Bucharest in 2012.

She is currently an Assistant at the Department of Electrical, Power Systems and Aerospace Engineering, University of Craiova. Her research activities include modeling and numerical simulation of electric and magnetic fields and analysis of electrical circuits.

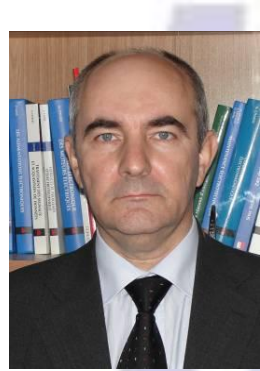

Lucian Mandache received the M.S. degree in electrical engineering from the University of Craiova, Romania in 1987, and the Ph.D. degree from the Politehnica University of Bucharest, in 2003. He joined the R\&D and Testing National Institute for Electrical Engineering (ICMET) Craiova, in 1989, leading many research and design projects for LV equipment of power conversion and unconventional measurement systems. In 1998, he joined the Department of Fundamentals in Electrical Engineering, Faculty of Electrical Engineering, University of Craiova, where he is currently Professor. His current research interests include nonlinear and switched-mode analog circuit analysis, modeling and simulation of ferromagnetic core devices, power quality diagnosis and improvement solutions, miscellaneous CAD/CAE tools. 